\title{
Comparação entre modelo DEA clusterizado pela escala de operação e modelo DEA tradicional para retornos variáveis de escala na avaliação de empresas aéreas brasileiras
}

\author{
Ana Paula dos Santos Rubem ${ }^{1}$, Ariane Lima de Moura², João Carlos Correia Baptista Soares de Mello ${ }^{3}$ \\ e Bruno Machado Fernandes dos Santos ${ }^{4}$
}

\begin{abstract}
Resumo: O objetivo deste artigo é realizar uma análise comparativa dos resultados de eficiência obtidos usando uma abordagem tradicional de Análise Envoltória de Dados (DEA, do inglês Data Envelopment Analysis) em contraposição à metodologia aqui proposta, que representa uma forma alternativa de lidar com diferenças na escala de operação das unidades produtivas. Para tanto, é conduzido um estudo de caso sobre o desempenho operacional das empresas brasileiras de transporte aéreo de passageiros, quanto ao uso da frota. A proposta combina clusterização das unidades produtivas segundo a escala de operação, aplicação do modelo DEA original para retornos constantes de escala separadamente em cada cluster, além da compensação das diferenças de escala entre os clusters. Os resultados foram comparados aos do modelo DEA tradicional para retornos variáveis de escala, tendo revelado que a abordagem proposta aumentou a discriminação entre as unidades, sem grande impacto nas ordenações finais.

Palavras-chave: Transporte Aéreo; Análise Envoltória de Dados; Clusterização.
\end{abstract}

\begin{abstract}
This paper performs a comparative analysis of the relative efficiency results obtained using a traditional approach of Data Envelopment Analysis in contrast to the methodology proposed here, which comprises an alternative way of dealing with differences in scale of operation of production units. For that, we will conduct a case study on the operational performance of Brazilian passenger airlines, regarding the use of the fleet. The proposal combines clustering of production units according to their scale of operation, original DEA model for constant returns to scale applied separately in each cluster, and compensation of differences in scale among clusters. We compared the results to traditional DEA model for variable returnsto-scale, which revealed that the proposed approach increased the discrimination among units without major impact on the final rankings.
\end{abstract}

Keywords: Air Transport; Data Envelopment Analysis; Clustering.

\section{INTRODUÇÃO}

Desde a década de 1990, o setor de transporte aéreo brasileiro sofreu mudanças significativas, tendo assistido a um processo de desregulamentação, que deu fim à política de controle de mercado implantada nos anos 1960 (Lima et al., 2011). O processo teve início com o fim dos monopólios regionais, o que incentivou o acesso de novas empresas e a disputa de preços, com a definição de faixas tarifárias. Em 2001, deu-se a liberalização total dos preços, além da maior flexibilidade nos processos de entrada de empresas e pedidos de novas rotas aéreas, frequências de voo e aeronaves, o que acarretou, inclusive, o surgimento da Gol, a primeira companhia de baixo custo do país (Evangelho et al., 2005). Os primeiros sinais de diminuição na competição foram notados por ocasião dos voos compartilhados entre a Varig e a TAM (Soares de Mello et al., 2009). A tendência se acentuou em 2006, com as dificuldades enfrentadas pela Varig, então líder de mercado, que acabou adquirida pela Gol, em 2008. A partir daí, teve início uma fase de concentração,

\footnotetext{
1 Ana Paula dos Santos Rubem, Departamento de Engenharia de Produção. Universidade Federal Fluminense (anarubem@id.uff.br)

2 Ariane Lima de Moura, Departamento de Engenharia de Produção.

Universidade Federal Fluminense. (ariane.moura@yahoo.com.br)

3 João Carlos Correia Baptista Soares de Mello, Departamento de

Engenharia de Produção. Universidade Federal Fluminense.

(jcsmello@producao.uff.br)

${ }^{4}$ Bruno Machado Fernandes dos Santos, Departamento de Engenharia de Produção. Universidade Federal Fluminense.

(brunomac_20@hotmail.com)
}

Manuscrito recebido em 21/08/2015 e aprovado para publicação em 24/09/2015.

Este artigo é parte de TRANSPORTES v. 23, n. 4, 2015. ISSN: 2237-1346 (online). DOI:10.14295/transportes.v23i4.984

TRANSPORTES v. 23, n. 4 (2015), p. 21-30 que levou à instalação de um quase-duopólio. Contudo, nos últimos anos, as empresas líderes (Gol/Varig e TAM) têm apresentado resultados ruins, enquanto a Azul vem crescendo consideravelmente.

$\mathrm{Na}$ literatura recente, grande parte dos estudos que usam Análise Envoltória de Dados (DEA, de Data Envelopment Analysis) na avaliação da eficiência das empresas aéreas brasileiras aplica o modelo BCC (Banker et al., 1984), em razão da acentuada diferença entre as escalas de operação das companhias (e.g., Soares de Mello et al., 2003; Correia et al., 2011; Silveira et al., 2012a, 2012b). A alegação é que, sob tais condições, não se deve negligenciar os efeitos de escala, o que impede o uso do modelo DEA original (CCR: Charnes et al., 1978), que supõe retornos constantes de escala (CRS, de Constant Returns-to-Scale). Contudo, o modelo BCC, que assume retornos variáveis de escala (VRS, de Variable Returns-to-Scale), apresenta certas desvantagens. Nesse sentido, a metodologia aqui proposta representa uma alternativa ao uso do modelo BCC, uma vez que busca lidar com as diferenças na escala de operação das unidades produtivas, de forma diferente da usualmente empregada. A proposta combina clusterização exante, por meio de um método baseado no K-means (Steinhaus, 1957; MacQueen, 1967), com a aplicação do modelo CCR, seguida pela compensação entre clusters, apresentada em Gomes et al. (2012b). O que se observa, comumente na literatura, é a aplicação de técnicas de compensação para tratar a não-homogeneidade das unidades produtivas. Desse modo, além da proposta metodológica em si, outro aspecto inovador deste trabalho reside em adaptar o uso de tais técnicas para o tratamento de diferenças de escala.

Em síntese, o objetivo deste artigo é realizar uma análise comparativa dos resultados de eficiência obtidos com o 
uso da metodologia proposta em contraposição aos da abordagem BCC tradicional. Para tanto, será conduzido um estudo de caso sobre o desempenho operacional das empresas brasileiras de transporte aéreo de passageiros, no período de 2007 a 2010, no que se refere, especificamente, ao uso da frota.

Dentre as empresas avaliadas, há algumas estritamente regionais, que operam uma pequena frota de aeronaves com baixa capacidade de transporte (e.g., Abaeté, Meta, Sol). Outras que, embora cubram todo o país, operam apenas voos domésticos, usando uma frota composta por um número maior de aeronaves com capacidade de transporte intermediário (e.g., Avianca, Azul, Trip, Webjet). Há, ainda, empresas que operam rotas nacionais e internacionais, utilizando uma extensa frota formada por modelos com grande capacidade de transporte (e.g., Gol/Varig, TAM). Portanto, não parece razoável supor que as companhias analisadas operem sob as mesmas condições de escala.

O restante do artigo está organizado da seguinte forma: a Seção 2 contém uma visão geral da metodologia proposta; a Seção 3 descreve a modelagem do problema e apresenta os principais resultados; e a Seção 4 traz as conclusões e sugestões para trabalhos futuros.

\section{REFERENCIAL TEÓRICO}

\subsection{DEA: Análise Envoltória de Dados}

DEA é uma abordagem não-paramétrica baseada em programação matemática, cujo objetivo é calcular a eficiência das unidades produtivas (DMUs, de Decision Making Units), as quais são otimizadas individualmente, comparando os recursos utilizados (inputs) e as quantidades produzidas (outputs) aos níveis das demais unidades. O resultado é a construção de uma fronteira eficiente. Apenas as DMUs nela situadas são consideradas eficientes (pontuação igual a 100\%). Para as ineficientes, são calculados alvos e um conjunto de referência. Os alvos são os níveis de inputs e outputs que devem ser atingidos para que se tornem eficientes. O conjunto de referência representa as DMUs eficientes (benchmarks) que servem como exemplos de boas práticas de gestão.

Há dois modelos tradicionais: os já mencionados CCR e BCC. O primeiro assume a hipótese CRS, ou seja, qualquer variação nos inputs produz uma variação proporcional nos outputs. O segundo, desenvolvido sob a suposição VRS, substitui o axioma da proporcionalidade entre inputs e outputs pelo da convexidade. Tais modelos possuem formulações duais (envelope e multiplicadores), que fornecem o mesmo resultado de eficiência. Tradicionalmente, há duas orientações radiais possíveis para esses modelos: a input, que busca minimizar os recursos sem alterar os níveis de produção; e a output, que visa maximizar a produção, sem alterar a quantidade de recursos utilizados. Assim, considerando um processo produtivo em que cada $\operatorname{DMU}_{k}(k=$ $1, \ldots, n)$ utiliza $r$ inputs $x_{i k}(i=1, \ldots, r)$ para produzir $s$ output $y_{j k}(j=1, \ldots, s)$, o modelo CCR orientado a inputs, na formulação do envelope, é dado por:

Min $h_{o}$

sujeito a

$$
\begin{aligned}
& h_{o} x_{i o} \geq \sum_{k} \lambda_{k} x_{i k}, \forall i \\
& \sum_{k} \lambda_{k} y_{j k} \geq y_{j o}, \forall j \\
& \lambda_{k} \geq 0, \forall k
\end{aligned}
$$

onde $h_{o}$ é a eficiência da unidade em análise $\left(\mathrm{DMU}_{o}\right) ; x_{i o}$ e $y_{j o}$ são, respectivamente, os inputs e outputs da $\mathrm{DMU}_{o}$; e $\lambda_{k}$ é a contribuição individual da $\mathrm{DMU}_{k}$ na definição do alvo da $\mathrm{DMU}_{o}$. As variáveis de decisão são $h_{o}$ e $\left\{\lambda_{k}\right\}$. Nessa formulação, o modelo BCC é obtido pela simples adição da restrição de convexidade: $\sum_{k} \lambda_{k}=1$.

Embora se proponha a permitir que uma DMU possa ser eficiente, independente da escala em que opera, o modelo BCC apresenta alguns inconvenientes: por exemplo, classificação inapropriada de DMUs como eficientes (Ali, 1993); elevação nas pontuações de eficiência, muitas vezes, reduzindo a capacidade de discriminação, em razão do aumento do número de unidades eficientes; obtenção de pontuações distintas, dependendo da orientação adotada; eficiências negativas implícitas (Soares de Mello et al., 2013; Gomes Júnior et al., 2013). Portanto, para evitar o uso do modelo BCC, mas ainda assim assegurar o tratamento das diferenças de escala de operação necessário à aplicação do modelo CCR, optou-se por uma abordagem alternativa, que combina clusterização ex-ante e compensação ex-post.

\subsection{Clusterização em DEA}

De modo geral, a análise de cluster é uma metodologia que compreende diferentes técnicas destinadas a avaliar a semelhança entre as unidades de um conjunto, segundo critérios predefinidos, a fim de formar grupos de similaridade. Neste artigo, tais técnicas são usadas para identificar grupos de empresas aéreas que operam sob condições comparáveis de escala.

Em geral, as abordagens que combinam DEA e técnicas de clusterização, inicialmente (ex-ante), agrupam as DMUs, de acordo com critérios estruturais ou ambientais prefixados, para, então, aplicar o modelo CCR separadamente para cada grupo formado. Aqui, ao invés de variáveis exógenas, o próprio input (capacidade da frota) do processo será usado na definição dos agrupamentos. Alternativamente, é possível adotar a abordagem inversa (ex-post), em que se calcula a eficiência de todas as unidades do conjunto de análise usando o modelo CCR para, só então, agrupá-las, segundo a pontuação obtida (Soares de Mello et al., 2012).

$\mathrm{O}$ primeiro tipo de abordagem (ex-ante) resulta em grupos disjuntos (interseção nula). Em DEA, isso inviabiliza a comparação direta entre os resultados de eficiência de DMUs pertencentes a clusters diferentes, pois a eficiência de uma DMU só pode ser comparada àquelas inseridas no mesmo subconjunto de análise. A abordagem ex-ante aqui proposta lida com essa questão, usando uma solução de compensação, detalhada mais adiante.

Neste artigo, primeiramente, os clusters são definidos, a partir de critérios associados à escala de operação das DMUs (capacidade da frota). Em seguida, é aplicada uma 


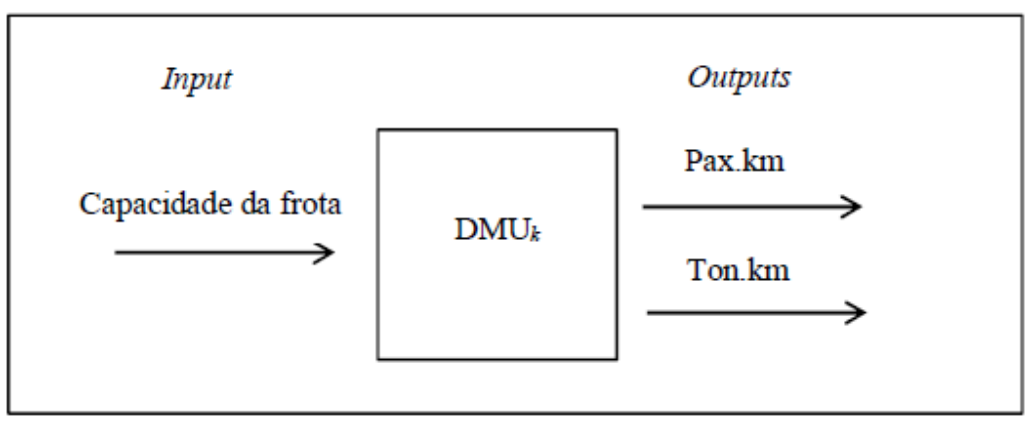

Figura 1. Representação do processo produtivo da $\mathrm{DMU}_{\mathrm{k}}$

técnica de compensação, originalmente proposta para ajustar a não-homogeneidade das DMUs, mas aqui adaptada para tratar diferenças de escala. Assim, a aplicação dessa técnica busca ajustar (ou compensar) os resultados dos clusters entre si e, por conseguinte, das DMUs pertencentes a eles, quanto à escala de operação, de modo a permitir a comparação direta entre os resultados de eficiência de todas DMUs do conjunto de análise, a despeito dos clusters serem disjuntos.

Para a compensação das diferenças entre clusters, é possível adotar uma solução $e x$-ante, que aplica um fator de correção às DMUs, antes de rodar o modelo DEA com todas as DMUs do conjunto de análise (e.g., Bertoloto e Soares de Mello 2011); ou ex-post, que calcula a eficiência usando um modelo DEA com todas as DMUs em conjunto para, a seguir, aplicar um fator de correção específico de cada cluster diretamente às eficiências calculadas, de forma a possibilitar a comparação global (Gomes et al., 2012b). Aqui, adota-se uma solução ex-post, que obedece aos passos abaixo relacionados.

1. Separar as DMUs em clusters de similaridade, conforme a escala de operação;

2. Rodar um modelo CCR, separadamente, para cada cluster e selecionar as DMUs eficientes;

3. Rodar um modelo CCR apenas com as DMUs eficientes de cada cluster, selecionadas no passo 2;

4. Calcular as eficiências médias das unidades do passo 3, separadas em seus clusters originais. Como essas DMUs eram eficientes nos seus clusters originais, o fato de a eficiência média não ser unitária pode ser atribuído a desvantagens de escala, e não necessariamente a ineficiências intrínsecas da DMU;

5. Rodar um modelo CCR com todas as DMUs do conjunto de análise; e

6. Usar as eficiências médias do passo 4 como fator de correção das pontuações de eficiência do cluster, dividindo-se a eficiência encontrada no passo 5 pela eficiência média do passo 4 . No caso de alguma eficiência corrigida ser maior que um, normalizar todas as pontuações de eficiências corrigidas, dividindo-as pelo valor máximo obtido.

As pontuações de eficiência finais são as do passo 6 . Diferentemente de Gomes et al. (2012b) e Bertoloto e Soares de Mello (2011), em que o agrupamento das DMUs é imediato, aqui, faz-se uso de um ferramental matemático. Dentre as várias técnicas disponíveis, optou-se pelo $K$-means (Steinhaus, 1957; MacQueen, 1967), um dos algoritmos de clusterização mais simples e referenciados (Jain, 2010).
Seu objetivo é particionar um conjunto em $k$ subconjuntos disjuntos, de forma que a soma dos quadrados das distâncias das unidades até o centro dos clusters seja minimizada. $\mathrm{O}$ valor de $k$ (o número de clusters desejados) é definido a priori e serve de entrada para o algoritmo, que segue as etapas relacionadas a seguir.

1. Começar com $k$ clusters, cada qual consistindo de uma única unidade (escolhida aleatoriamente dentre as observações), a qual denota o centro do respectivo cluster;

2. Adicionar novas unidades ao cluster cujo centro (ou média) estiver mais próximo da unidade adicionada;

3. Quando todas as $n$ unidades tiverem sido alocadas, recalcular as médias (centros) dos $k$ clusters; e

4. Repetir as etapas 2 e 3 até que os centros dos $k$ clusters não se alterem.

$\mathrm{O}$ algoritmo do $K$-means não, necessariamente, converge para o mínimo global. Além disso, o resultado pode ser bastante sensível aos valores iniciais (etapa 1). Mas, como será visto na Subseção 3.3, aqui, a maior dificuldade está relacionada ao tamanho dos clusters. Essa é uma limitação usual das abordagens que combinam DEA e clusterização sistemática. Propôs-se, então, um refinamento metodológico, detalhado na Subseção 3.3, que consiste em aglutinar clusters subsequentes gerados pelo $K$-means, até que se atinja um número mínimo de unidades em cada um deles.

\section{MODELAGEM E RESULTADOS}

\subsection{Definição das variáveis}

Em transporte aéreo, a otimização da frota evita a ociosidade das aeronaves (um dos principais fatores de produção). Isso contribui para a redução dos custos operacionais e, portanto, para o aumento dos lucros da empresa. Assim sendo, por simplicidade, supõe-se que cada companhia aérea de passageiros conduz um processo produtivo que engloba um input (capacidade da frota) e dois outputs (pax.km transportado e ton.km transportado). A Figura 1 ilustra a configuração adotada. Correia et al. (2011) usaram a mesma estrutura de variáveis, embora tenham aplicado um modelo Fuzzy-DEA. Gomes Júnior et al. (2015) também fizeram uso dessa mesma configuração para o processo produtivo, mas aplicaram uma abordagem DEA não radial.

Como cada empresa usa modelos de aeronaves distintos, com diferente capacidade de transporte, o input "capacidade da frota" denota a soma dos pesos máximos de decolagem de todas as aeronaves da frota, tal como descrito 
por Correia et al. (2011). Desse modo, o input engloba o tanto o tamanho da frota como sua respectiva capacidade de transporte (passageiros e carga).

Os outputs devem estar associados ao serviço oferecido pelas empresas, portanto, neste artigo, considera-se a quantidade total de passageiros transportados multiplicada pelo número total de quilômetros voados e a quantidade total de toneladas transportadas multiplicada pelo número total de quilômetros voados, respectivamente, "pax.km transportado" e "ton.km transportado".

Outros exemplos de configuração de inputs em problemas similares, mas com propósitos distintos do deste artigo, podem ser encontrados em Soares de Mello et al. (2003) e Silveira et al. (2012a, 2012b). O primeiro incorporou inputs adicionais afetos a combustível e mão-de-obra, enquanto os dois últimos acrescentaram apenas mão-deobra. Todavia, aqui, optou-se por uma estrutura mais simples, pois o objetivo é avaliar, especificamente, o uso da frota.

Assim, em termos gerais, com a estruturação de variáveis adotada busca-se mensurar a capacidade da empresa de usar a frota que possui de forma eficiente. A orientação a input foi usada, uma vez que o propósito é identificar as empresas que podem reduzir a capacidade de transporte de sua frota, sem prejuízo ao total de passageiros e carga transportados.

\subsection{Base de dados}

Os dados referem-se ao período de 2007 a 2010, e foram extraídos dos Anuários Estatísticos do Transporte Aéreo, disponíveis no site da Agência Nacional de Aviação Civil (ANAC, 2007, 2008, 2009, 2010). A análise começa em 2007, por ser o ano subsequente à grave crise no setor aéreo nacional, causada, principalmente, pela falência da Varig e pela queda do avião da Gol. Portanto, para que houvesse uma maior consistência nos dados, optou-se por analisar anos com características similares. Os dados referentes a 2011 e 2012 não contêm todas as variáveis consideradas e, por este motivo, não constam da análise, que termina em 2010.

Como a regulação e estrutura do setor aéreo nacional permaneceram praticamente inalteradas no período analisado, aplicou-se uma abordagem contemporânea, em que todas as observações são inseridas em conjunto na análise, considerando a mesma empresa como uma DMU independente em anos distintos. Essa é uma das formas de se aumentar o número de DMUs (Podinovski e Thanassoulis, 2007), que permite, ainda, a análise temporal (e.g., Soares de Mello et al., 2003; Correia et al., 2011; Gomes Júnior et al., 2015).

Devido à agregação de passageiros e carga em quilômetros transportados ao longo do ano, os dados referem-se à posição no final de cada ano. $\mathrm{O}$ mesmo ocorre com os dados da frota, embora ela possa variar durante o ano. Portanto, para estimar a posição da frota no fim do primeiro semestre, foram calculadas as médias, incorporando os valores praticados no ano anterior, bem como no ano em questão, as quais, em seguida, foram usadas no cálculo da capacidade da frota de cada DMU.
No período em análise, houve uma média anual de 20 companhias aéreas de transporte de passageiros em operação, o que corresponde a 78 DMUs. Dentre elas, cinco foram descartadas, tendo em vista a inexistência de informações de frota. A Tabela 1 apresenta os valores de input $\mathrm{e}$ outputs praticados pelas 73 DMUs avaliadas.

\subsection{Abordagem proposta: clusterização seguida de compensação de escala}

Como descrito na Subseção 2.2, na geração dos clusters foi usado o método $K$-means, aplicado à variável de input "capacidade da frota", uma vez que o objetivo é criar grupos cujas DMUs neles inseridas operem sob condições similares de escala.

Para implementação do K-means, foi usado o software XLSTAT, versão 2014.3.01. Contudo, observou-se que, ao se inicializar o algoritmo com valores de $k \in[2,8]$, eram gerados clusters compostos por menos de nove DMUs, valor este que aqui denota a quantidade mínima para que se obtenha resultados de eficiência confiáveis. Isso porque, segundo Banker et al. (1989), o total de DMUs em cada cluster deve ser o triplo do número de inputs e outputs usados na modelagem.

Considerando esse intervalo de variação para $k$, os clusters com quantidade insuficiente de DMUs (i.e., inferior a nove) abrangiam empresas com capacidade de frota variando entre grande e intermediária. Já as DMUs de menor capacidade de frota eram agrupadas em um cluster único, cuja composição variava entre 32 e 64 empresas, e a capacidade média da frota (centro do cluster) variava entre 25,1 e 308,3 toneladas.

Buscando superar tal condição, primeiro, definiu-se que o valor de $k$ a ser usado como entrada seria igual a quatro, considerando-se que, caso houvesse equilíbrio na divisão das DMUs entre os clusters, $k$ deveria ser menor ou igual a oito, para garantir o tamanho mínimo recomendável para cada cluster. Após alguns testes, verificou-se que, para $k>4$, eram gerados clusters compostos por uma única DMU cada, os quais mesmo agregados, ainda resultariam em clusters com menos de nove DMUs.

Por essa razão, a estratégia adotada consistiu em rodar o algoritmo K-means $(\mathrm{k}=4)$ e agregar os clusters de tamanho inferior a nove, respeitando-se a ordenação das escalas de operação das DMUs neles inseridas, isto é, os centros dos clusters a serem agrupados deveriam ser subsequentes.

Formou-se, então, o primeiro cluster. As DMUs pertencentes a ele foram retiradas, e o algoritmo $K$-means $(k=4)$ foi rodado mais uma vez. $O$ processo foi repetido até que todas as DMUs do conjunto de análise fossem alocadas a algum cluster (todos com no mínimo nove DMUs), tendo resultado na formação dos cinco clusters exibidos na Tabela 2.

Nota-se que certas DMUs situadas no limite de seus clusters apresentam capacidade de transporte da frota (input) mais próxima de uma DMU de outro cluster do que de outra de seu próprio cluster: por exemplo, a escala de operação da empresa Team em 2008 (cluster 1) se assemelha mais à praticada pela Sete no mesmo ano (cluster 2), do que à da Sol em 2009 (cluster 1). Isso é problema usual das 
Comparação entre modelo DEA clusterizado pela escala de operação e modelo DEA

tradicional para retornos variáveis de escala na avaliação de empresas aéreas brasileiras

Tabela 1. Valores de input e outputs praticados pelas DMUs avaliadas

\begin{tabular}{|c|c|c|c|c|c|c|c|}
\hline$D M U$ & $\begin{array}{l}\text { Capacidade } \\
\text { da frota }(t)\end{array}$ & $\begin{array}{l}\text { Pax.km } \\
\text { (milhares) }\end{array}$ & $\begin{array}{l}\text { Ton.km } \\
\text { (milhares) }\end{array}$ & $D M U$ & $\begin{array}{l}\text { Capacidade } \\
\text { da frota }(t)\end{array}$ & $\begin{array}{l}\text { Pax.km } \\
\text { (milhares) }\end{array}$ & $\begin{array}{l}\text { Ton.km } \\
\text { (milhares) }\end{array}$ \\
\hline Abaete_10 & 11 & 1934 & 161 & Abaete_08 & 12 & 1.378 & 125 \\
\hline Avianca_10 & 789 & 1.856 .243 & 171.288 & Air Minas_08 & 32 & 18.744 & 1.680 \\
\hline Azul_10 & 1.041 & 4.306 .850 & 357.500 & Gol/Varig_08 & 8.658 & 26.296 .872 & 2.753 .740 \\
\hline Gol/Varig_10 & 9.191 & 31.402 .872 & 2.917 .360 & Meta_08 & 30 & 37.800 & 3.984 \\
\hline Meta_10 & 27 & 20.088 & 1.787 & NHT_08 & 34 & 16.678 & 1.499 \\
\hline $\mathrm{NHT}_{-} 10$ & 40 & 10.425 & 874 & Oceanair_08 & 1.116 & 1.464 .627 & 159.498 \\
\hline Noar_10 & 10 & 5.005 & 1.426 & Pantanal_08 & 101 & 79.126 & 7.296 \\
\hline Pantanal_10 & 205 & 242.946 & 20.823 & Passaredo_08 & 54 & 86.746 & 8.532 \\
\hline Passaredo_10 & 215 & 428.593 & 37.401 & Puma_08 & 28 & 4.172 & 434 \\
\hline Puma_10 & 59 & 86.804 & 45.216 & Rico_08 & 124 & 50.013 & 5.252 \\
\hline Rico_10 & 182 & 634 & 51.767 & Sete_08 & 20 & 9.075 & 1.000 \\
\hline Sete_10 & 29 & 16.795 & 1.619 & TAF_08 & 432 & 77.986 & 44.866 \\
\hline Sol_10 & 7 & 2.245 & 186 & TAM_08 & 12.541 & 40.702 .300 & 4.671 .559 \\
\hline TAM_10 & 15.114 & 51.712 .453 & 5.010 .977 & Team_08 & 18 & 3.480 & 287 \\
\hline Team_10 & 17 & 3.084 & 238 & Total_08 & 518 & 66.507 & 41.849 \\
\hline Total_10 & 542 & 61.992 & 52.740 & Trip_08 & 281 & 517.235 & 51.882 \\
\hline Trip_10 & 824 & 1.547 .564 & 136.879 & Webjet_08 & 431 & 1.180 .795 & 123.580 \\
\hline Webjet_10 & 1.322 & 4.130 .647 & 360.628 & & & & \\
\hline Abaete_09 & 12 & 1.645 & 139 & Abaete_07 & 15 & 2.492 & 227 \\
\hline AirMinas_09 & 43 & 20.820 & 1.726 & AirMinas_07 & 32 & 12.289 & 1.160 \\
\hline Avianca_09 & 829 & 1.438 .350 & 131.355 & Gol_07 & 4.689 & 20.306 .538 & 2.119 .062 \\
\hline Azul_09 & 391 & 2.110 .707 & 176.730 & Mega_07 & 4 & 1.072 & 313 \\
\hline Gol/Varig_09 & 8.774 & 26.339 .094 & 2.391.779 & Meta_07 & 30 & 24.602 & 2.706 \\
\hline Meta_09 & 30 & 20.277 & 1.820 & NHT_07 & 28 & 12.638 & 1.127 \\
\hline NHT_09 & 40 & 15.435 & 1.278 & Oceanair_07 & 918 & 1.079 .481 & 120.141 \\
\hline Pantanal_09 & 92 & 77.465 & 6.544 & Pantanal_07 & 102 & 93.420 & 8.679 \\
\hline Passaredo_09 & 95 & 221.821 & 19.284 & Passaredo_07 & 30 & 47.629 & 4.726 \\
\hline Puma_09 & 28 & 43 & 4 & Puma_07 & 28 & 9.763 & 1.013 \\
\hline Rico_09 & 173 & 4.270 & 366 & Rico_07 & 171 & 161.617 & 17.833 \\
\hline Sete_09 & 23 & 10.514 & 1.044 & Sete_07 & 20 & 8.057 & 873 \\
\hline Sol_09 & 3 & 1.212 & 99 & TAF_07 & 399 & 167.685 & 51.366 \\
\hline TAF_09 & 159 & 4.826 & 37.645 & TAM_07 & 9.553 & 33.445 .234 & 3.806 .729 \\
\hline TAM_09 & 14.028 & 44.570 .946 & 4.341 .646 & Team_07 & 21 & 3.087 & 262 \\
\hline Team_09 & 14 & 3.476 & 268 & Total_07 & 510 & 225.952 & 49.665 \\
\hline Total_09 & 498 & 62.230 & 49.086 & Trip_07 & 157 & 208.626 & 22.677 \\
\hline Trip_09 & 512 & 851.302 & 76.279 & Varig_07 & 6.597 & 3.831 .335 & 478.611 \\
\hline Webjet_09 & 955 & 2.522 .478 & 226.507 & Webjet_07 & 155 & 345.579 & 35.885 \\
\hline
\end{tabular}

abordagens que combinam clusterização sistemática e DEA.

Após a formação dos clusters, aplicou-se o modelo CCR orientado a input em (1), considerando-se as variáveis definidas na Subseção 3.1, separadamente para cada cluster (passo 2 da solução de compensação descrita na Subseção 2.2). No cálculo das eficiências, foi usado o software SIAD (Angulo-Meza et al., 2005), versão 3.0. Os resultados constam da Tabela 3.

Na Tabela 3, observa-se que, ao todo, sete DMUs foram eficientes: uma no cluster 1 (Noar, em 2010); duas no cluster 2 (Passaredo, em 2008; e a Puma, em 2010); duas no cluster 3 (Passaredo, em 2009; e Rico, em 2010); uma no cluster 4 (Azul, em 2009); e uma no cluster 5 (Gol, em 2007).

A seguir, as DMUs eficientes de cada cluster foram separadas e o mesmo modelo CCR usado anteriormente foi aplicado a estas DMUs (passo 3). A partir dos resultados de eficiência obtidos, calculou-se, então, as eficiências médias das DMUs do cluster de eficientes, levando-se em conta seus clusters originais (passo 4). Os resultados constam da Tabela 4.

Na Tabela 4, o fato de apenas a empresa Azul, em 2009 , ter sido $100 \%$ eficiente no cluster de eficientes sugere TRANSPORTES v. 23, n. 4 (2015), p. 21-30 que o cluster 4 é o único operando em escala ótima, enquanto os demais apresentam desvantagens de escala que têm impacto negativo na eficiência, a despeito de quaisquer outras ineficiências gerenciais.

A etapa seguinte (passo 5) consiste em aplicar o mesmo modelo CCR a todas as DMUs do conjunto de análise, aqui chamado de modelo geral. Em seguida (passo 6), usou-se as eficiências médias de cada cluster (vide Tabela 4), como fator de correção das eficiências gerais, dividindose as eficiências obtidas no modelo geral, pelas eficiências médias de cada cluster, considerando-se o cluster a que as DMUs foram originalmente designadas. Os resultados foram, então, normalizados.

A Tabela 5 apresenta os resultados de eficiência sob o modelo geral, bem como as respectivas eficiências corrigidas e normalizadas. Após a compensação e subsequente normalização, a única DMU que se manteve $100 \%$ eficiente foi a empresa Puma, em 2010, originalmente alocada ao cluster 2.

\subsection{Comparação com modelo BCC tradicional}

Para fins comparativos, o modelo BCC tradicional, orientado a input, comumente utilizado quando não se pode 
RUBEM, A.P.S; MOURA, A.L; SOARES DE MELLO, J.C.C.B; SANTOS, B.M.F.

Tabela 2. Composição dos clusters gerados, usando o input "capacidade da frota"

\begin{tabular}{|c|c|c|c|c|c|c|c|c|c|}
\hline \multicolumn{2}{|c|}{ Cluster $1(n=11)$} & \multicolumn{2}{|c|}{ Cluster $2(n=22)$} & \multicolumn{2}{|c|}{ Cluster $3(n=13)$} & \multicolumn{2}{|c|}{ Cluster $4(n=18)$} & \multicolumn{2}{|l|}{ Cluster $5(n=9)$} \\
\hline$D M U$ & Input & $\overline{D M U}$ & Input & $D M U$ & Input & $D M U$ & Input & $D M U$ & Input \\
\hline Sol_09 & 3,3 & Sete_08 & 19,9 & Pantanal_09 & 91,9 & Trip_08 & 280,7 & Gol_07 & $4.688,5$ \\
\hline Mega_07 & 4,0 & Sete_07 & 20,0 & Passaredo_09 & 95,3 & Azul_09 & 391,4 & Varig_07 & $6.597,0$ \\
\hline Sol_10 & 6,6 & Team_07 & 21,0 & Pantanal_08 & 101,1 & TAF_07 & 399,0 & Gol/Varig_08 & $8.658,4$ \\
\hline Noar_10 & 9,9 & Sete_09 & 23,1 & Pantanal_07 & 102,0 & Webjet_08 & 431,3 & Gol/Varig_09 & $8.774,1$ \\
\hline Abaete_10 & 11,2 & Meta_10 & 27,4 & Rico_08 & 124,1 & TAF_08 & 432,3 & Gol/Varig_10 & $9.190,5$ \\
\hline Abaete_09 & 11,5 & Puma_09 & 28,0 & Webjet_07 & 155,0 & Total_09 & 497,8 & TAM_07 & $9.553,3$ \\
\hline Abaete_08 & 11,9 & Puma_08 & 28,0 & Trip_07 & 157,0 & Total_07 & 510,0 & TAM_08 & $12.541,3$ \\
\hline Team_09 & 13,6 & NHT_07 & 28,0 & TAF_09 & 158,9 & Trip_09 & 511,7 & TAM_09 & $14.028,3$ \\
\hline Abaete_07 & 15,0 & Puma_07 & 28,0 & Rico_07 & 171,3 & Total_08 & 517,8 & TAM_10 & $15.113,5$ \\
\hline Team 10 & 16,5 & Sete 10 & 29,1 & Rico 09 & 173,3 & Total 10 & 542,0 & & \\
\hline Team_08 & 17,5 & Meta_09 & 29,7 & Rico_10 & 182,2 & Avianca_10 & 788,5 & & \\
\hline & & Meta_08 & 29,8 & Pantanal_10 & 204,5 & Trip_10 & 824,0 & & \\
\hline & & Meta_07 & 30,0 & Passaredo_10 & 215,2 & Avianca_09 & 828,6 & & \\
\hline & & Passaredo_07 & 30,0 & & & Oceanair_07 & 918,0 & & \\
\hline & & AirMinas_08 & 31,6 & & & Webjet_09 & 955,1 & & \\
\hline & & AirMinas_07 & 32,0 & & & Azul_10 & $1.040,9$ & & \\
\hline & & NHT_08 & 34,0 & & & Oceanair_08 & $1.115,9$ & & \\
\hline & & NHT_10 & 39,6 & & & Webjet_10 & $1.322,3$ & & \\
\hline & & NHT_09 & 39,6 & & & & & & \\
\hline & & AirMinas_09 & 43,4 & & & & & & \\
\hline & & Passaredo_08 & 53,8 & & & & & & \\
\hline & & Puma_10 & 58,5 & & & & & & \\
\hline
\end{tabular}

Tabela 3. Eficiências CCR em cada cluster

\begin{tabular}{|c|c|c|c|c|c|c|c|c|c|}
\hline \multicolumn{2}{|c|}{ Cluster 1} & \multicolumn{2}{|c|}{ Cluster 2} & \multicolumn{2}{|c|}{ Cluster 3} & \multicolumn{2}{|c|}{ Cluster 4} & \multicolumn{2}{|c|}{ Cluster 5} \\
\hline$D M U$ & Eficiência & $D M U$ & Eficiência & $D M U$ & Eficiência & $D M U$ & Eficiência & $D M U$ & Eficiência \\
\hline Sol_09 & $72,6 \%$ & Sete_08 & $28,3 \%$ & Pantanal_09 & $36,2 \%$ & Trip_08 & $40,9 \%$ & Gol_07 & $100,0 \%$ \\
\hline Mega_07 & $54,3 \%$ & Sete_07 & $25,0 \%$ & Passaredo_09 & $100,0 \%$ & Azul_09 & $100,0 \%$ & Varig_07 & $16,1 \%$ \\
\hline Sol_10 & $67,3 \%$ & Team_07 & $9,1 \%$ & Pantanal_08 & $34,1 \%$ & TAF_07 & $28,5 \%$ & Gol/Varig_08 & $70,4 \%$ \\
\hline Noar_10 & $100,0 \%$ & Sete_09 & $28,2 \%$ & Pantanal_07 & $40,0 \%$ & Webjet_08 & $63,5 \%$ & Gol/Varig_09 & $69,3 \%$ \\
\hline Abaete_10 & $34,2 \%$ & Meta_10 & $45,4 \%$ & Rico_08 & $18,2 \%$ & TAF_08 & $23,0 \%$ & Gol/Varig_10 & $78,9 \%$ \\
\hline Abaete_09 & $28,3 \%$ & Puma_09 & $0,1 \%$ & Webjet_07 & $100,0 \%$ & Total_09 & $21,8 \%$ & TAM_07 & $88,2 \%$ \\
\hline Abaete_08 & $22,9 \%$ & Puma_08 & $9,2 \%$ & Trip_07 & $61,9 \%$ & Total_07 & $21,6 \%$ & TAM_08 & $82,4 \%$ \\
\hline Team_09 & $50,6 \%$ & NHT_07 & $28,0 \%$ & TAF_09 & $83,6 \%$ & Trip_09 & $33,0 \%$ & TAM_09 & $73,4 \%$ \\
\hline Abaete_07 & $32,9 \%$ & Puma_07 & $21,6 \%$ & Rico_07 & $44,5 \%$ & Total_08 & $17,9 \%$ & TAM_10 & $79,0 \%$ \\
\hline Team_10 & $37,0 \%$ & Sete_10 & $35,7 \%$ & Rico_09 & $1,1 \%$ & Total_10 & $21,6 \%$ & & \\
\hline Team_08 & $39,3 \%$ & Meta_09 & $42,4 \%$ & Rico_10 & $100,0 \%$ & Avianca_10 & $48,1 \%$ & & \\
\hline & & Meta_08 & $78,7 \%$ & Pantanal_10 & $51,0 \%$ & Trip_10 & $36,8 \%$ & & \\
\hline & & Meta_07 & $51,0 \%$ & Passaredo_10 & $85,7 \%$ & Avianca_09 & $35,1 \%$ & & \\
\hline & & Passaredo_07 & $98,4 \%$ & & & Oceanair_07 & $29,0 \%$ & & \\
\hline & & AirMinas_08 & $36,8 \%$ & & & Webjet_09 & $52,5 \%$ & & \\
\hline & & AirMinas_07 & $23,8 \%$ & & & Azul_10 & $76,7 \%$ & & \\
\hline & & NHT_08 & $30,4 \%$ & & & Oceanair_08 & $31,7 \%$ & & \\
\hline & & NHT_10 & $16,3 \%$ & & & Webjet_10 & $60,4 \%$ & & \\
\hline & & NHT_09 & $24,2 \%$ & & & & & & \\
\hline & & AirMinas 09 & $29,7 \%$ & & & & & & \\
\hline & & Passaredo 08 & $100,0 \%$ & & & & & & \\
\hline & & Puma_10 & $100,0 \%$ & & & & & & \\
\hline
\end{tabular}

negligenciar as diferenças de escala, foi aplicado ao conjunto de 73 DMUs como um todo, considerando as variáveis definidas na Subseção 3.1. Os resultados de eficiência (normalizada) da abordagem aqui proposta (vide Tabela 5) e da abordagem BCC tradicional, bem como as respectivas ordenações, são exibidos na Tabela 6.

Os resultados indicam que a abordagem proposta discrimina melhor as DMUs, gerando apenas uma empresa eficiente, contra dez no modelo BCC tradicional. A única DMU eficiente nas duas abordagens foi a empresa Puma, em 2010. No modelo BCC tradicional, duas DMUs eficientes foram assim classificadas por terem usado o menor nível de recursos (Sol, em 2009) ou produzido a maior quantidade em, ao menos, um dos outputs (TAM, em 2010). De fato, essa classificação inadequada (mencionada na Subseção 2.1) é uma das desvantagens do modelo BCC, que levou à proposta de uma metodologia alternativa. $\mathrm{Na}$ abordagem alternativa, essas mesmas DMUs tiveram resultados bem inferiores: $50 \%$ para TAM, em 2010; e 22\% para Sol, em 2009. 
Tabela 4. Eficiências no cluster de eficientes e médias nos clusters de origem

\begin{tabular}{llll}
\hline Cluster & DMU & Eficiência & $\begin{array}{l}\text { Média no } \\
\text { cluster }\end{array}$ \\
\hline Cluster 1 & Noar_10 & $20,7 \%$ & 0,2074 \\
Cluster 2 & Passaredo_08 & $32,5 \%$ & 0,6627 \\
& Puma_10 & $100,0 \%$ & \\
& Passaredo_09 & $44,0 \%$ & \\
Cluster 3 & Webjet_07 & $46,4 \%$ & 0,4237 \\
& Rico_10 & $36,8 \%$ & \\
Cluster 4 & Azul_09 & $100,0 \%$ & 1,0000 \\
Cluster 5 & Gol_07 & $90,3 \%$ & 0,9030 \\
\hline
\end{tabular}

Tabela 5. Eficiências padrão sob o modelo CCR geral, eficiências compensadas pelo fator de correção dos clusters e eficiências compensadas normalizadas

\begin{tabular}{|c|c|c|c|c|c|c|c|c|c|}
\hline \multirow{2}{*}{ Cluster } & \multirow{2}{*}{$D M U$} & \multicolumn{3}{|c|}{ Eficiência } & \multirow{2}{*}{ Cluster } & \multirow{2}{*}{$D M U$} & \multicolumn{3}{|c|}{ Eficiência } \\
\hline & & Geral & Compensada & Normalizada & & & Geral & Compensada & Normalizada \\
\hline \multirow{13}{*}{ Cluster 1} & Sol_09 & $6,8 \%$ & $32,8 \%$ & $21,8 \%$ & \multirow{13}{*}{ Cluster 3} & Pantanal_09 & $15,7 \%$ & $37,1 \%$ & $24,6 \%$ \\
\hline & Mēga_07 & $11,2 \%$ & $54,0 \%$ & $35,8 \%$ & & Passaredo_09 & $44,0 \%$ & $103,8 \%$ & $68,8 \%$ \\
\hline & Sol_10 & $6,3 \%$ & $30,4 \%$ & $20,2 \%$ & & Pantanal_08 & $15,3 \%$ & $36,0 \%$ & $23,9 \%$ \\
\hline & Noar_10 & $20,7 \%$ & $100,0 \%$ & $66,3 \%$ & & Pantanal_07 & $17,9 \%$ & $42,3 \%$ & $28,0 \%$ \\
\hline & Abaete_10 & $3,2 \%$ & $15,4 \%$ & $10,2 \%$ & & Rico_08 & $8,4 \%$ & $19,9 \%$ & $13,2 \%$ \\
\hline & Abaete_09 & $2,7 \%$ & $12,9 \%$ & $8,5 \%$ & & Webjet_07 & $46,4 \%$ & $109,4 \%$ & $72,5 \%$ \\
\hline & Abaete_08 & $2,2 \%$ & $10,8 \%$ & $7,1 \%$ & & Trip_07 & $28,3 \%$ & $66,9 \%$ & $44,3 \%$ \\
\hline & Team_09 & $4,7 \%$ & $22,9 \%$ & $15,1 \%$ & & TAF_09 & $30,6 \%$ & $72,3 \%$ & $47,9 \%$ \\
\hline & Abaete_07 & $3,2 \%$ & $15,5 \%$ & $10,3 \%$ & & Rico_07 & $20,3 \%$ & $47,9 \%$ & $31,8 \%$ \\
\hline & Team_10 & $3,5 \%$ & $16,7 \%$ & $11,1 \%$ & & Rico_09 & $0,5 \%$ & $1,1 \%$ & $0,7 \%$ \\
\hline & \multirow[t]{3}{*}{ Team_08 } & \multirow[t]{3}{*}{$3,7 \%$} & \multirow[t]{3}{*}{$17,8 \%$} & \multirow[t]{3}{*}{$11,8 \%$} & & Rico_10 & $36,8 \%$ & $86,8 \%$ & $57,5 \%$ \\
\hline & & & & & & Pantanal_10 & $22,3 \%$ & $52,6 \%$ & $34,9 \%$ \\
\hline & & & & & & Passaredo_10 & $37,7 \%$ & $89,0 \%$ & $59,0 \%$ \\
\hline \multirow{31}{*}{ Cluster 2} & Sete_08 & $9,8 \%$ & $14,8 \%$ & $9,8 \%$ & \multirow{22}{*}{ Cluster 4} & Trip_08 & $37,6 \%$ & $37,6 \%$ & $24,9 \%$ \\
\hline & Sete_07 & $8,6 \%$ & $12,9 \%$ & $8,6 \%$ & & Azul_09 & $100,0 \%$ & $100,0 \%$ & $66,3 \%$ \\
\hline & Team_07 & $2,7 \%$ & $4,1 \%$ & $2,7 \%$ & & TAF_07 & $18,2 \%$ & $18,2 \%$ & $12,1 \%$ \\
\hline & Sete_09 & $9,2 \%$ & $13,9 \%$ & $9,2 \%$ & & Webjet_08 & $57,2 \%$ & $57,2 \%$ & $37,9 \%$ \\
\hline & Meta_10 & $14,0 \%$ & $21,1 \%$ & $14,0 \%$ & & TAF_08 & $15,6 \%$ & $15,6 \%$ & $10,4 \%$ \\
\hline & Puma_09 & $0,03 \%$ & $0,04 \%$ & $0,03 \%$ & & Total_09 & $20,3 \%$ & $20,3 \%$ & $13,4 \%$ \\
\hline & Puma_08 & $3,1 \%$ & $4,7 \%$ & $3,1 \%$ & & Total_07 & $15,0 \%$ & $15,0 \%$ & $9,9 \%$ \\
\hline & NHT_07 & $8,6 \%$ & $13,0 \%$ & $8,6 \%$ & & Trip_09 & $31,9 \%$ & $31,9 \%$ & $21,2 \%$ \\
\hline & Puma_07 & $7,2 \%$ & $10,9 \%$ & $7,2 \%$ & & Total_08 & $13,6 \%$ & $13,6 \%$ & $9,0 \%$ \\
\hline & Sete_10 & $11,5 \%$ & $17,4 \%$ & $11,5 \%$ & & Total_10 & $21,0 \%$ & $21,0 \%$ & $13,9 \%$ \\
\hline & Meta_09 & $13,1 \%$ & $19,8 \%$ & $13,1 \%$ & & Avianca_10 & $45,9 \%$ & $45,9 \%$ & $30,4 \%$ \\
\hline & Meta_08 & $26,6 \%$ & $40,1 \%$ & $26,6 \%$ & & Trip_10 & $35,8 \%$ & $35,8 \%$ & $23,7 \%$ \\
\hline & Meta_07 & $17,6 \%$ & $26,6 \%$ & $17,6 \%$ & & Avianca_09 & $33,7 \%$ & $33,7 \%$ & $22,3 \%$ \\
\hline & Passaredo_07 & $32,2 \%$ & $48,6 \%$ & $32,2 \%$ & & Oceanair_07 & $25,4 \%$ & $25,4 \%$ & $16,9 \%$ \\
\hline & AirMinas_08 & $11,4 \%$ & $17,2 \%$ & $11,4 \%$ & & Webjet_09 & $50,8 \%$ & $50,8 \%$ & $33,6 \%$ \\
\hline & AirMinas_07 & $7,6 \%$ & $11,4 \%$ & $7,6 \%$ & & Azul_10 & $76,7 \%$ & $76,7 \%$ & $50,8 \%$ \\
\hline & NHT_08 & $9,4 \%$ & $14,2 \%$ & $9,4 \%$ & & Oceanair_08 & $28,0 \%$ & $28,0 \%$ & $18,6 \%$ \\
\hline & NHT_10 & $4,9 \%$ & $7,4 \%$ & $4,9 \%$ & & Webjet_10 & $59,2 \%$ & $59,2 \%$ & $39,2 \%$ \\
\hline & NHT_09 & $7,2 \%$ & $10,9 \%$ & $7,2 \%$ & & & & & \\
\hline & AirMinas_09 & $8,9 \%$ & $13,4 \%$ & $8,9 \%$ & & & & & \\
\hline & Passaredo_08 & $32,5 \%$ & $49,1 \%$ & $32,5 \%$ & & & & & \\
\hline & \multirow[t]{10}{*}{ Puma_10 } & $100,0 \%$ & $150,9 \%$ & $100,0 \%$ & & & & & \\
\hline & & & & & & Gol_07 & $90,3 \%$ & $100,0 \%$ & $66,3 \%$ \\
\hline & & & & & & Varig_07 & $13,4 \%$ & $14,9 \%$ & $9,9 \%$ \\
\hline & & & & & & Gol/Varig_08 & $63,4 \%$ & $70,3 \%$ & $46,6 \%$ \\
\hline & & & & & & Gol/Varig_09 & $58,0 \%$ & $64,3 \%$ & $42,6 \%$ \\
\hline & & & & & Cluster 5 & Gol/Varig_10 & $66,9 \%$ & $74,0 \%$ & $49,1 \%$ \\
\hline & & & & & & TAM_07 & $76,7 \%$ & $84,9 \%$ & $56,3 \%$ \\
\hline & & & & & & TAM_08 & $71,4 \%$ & $79,1 \%$ & $52,4 \%$ \\
\hline & & & & & & TAM_09 & $63,8 \%$ & $70,6 \%$ & $46,8 \%$ \\
\hline & & & & & & TAM_10 & $68,5 \%$ & $75,8 \%$ & $50,3 \%$ \\
\hline
\end{tabular}

Na Tabela 6, nota-se, ainda, que, na maioria dos casos (60 das 73 DMUs), a metodologia proposta resultou em pontuações inferiores às observadas no modelo BCC. Dentre as exceções, estão 11 DMUs pertencentes ao cluster 3: Pantanal, nos anos de 2008, 2009 e 2010; Passaredo, em 2009 e 2010; Rico, nos anos de 2007, 2008 e 2010; TAF, em 2009; Trip, em 2007; e Webjet, em 2007. Outra exceção é a empresa Noar, em 2010, que compõe o cluster 1.

Em relação à ordenação final os resultados das duas abordagens são bastante similares. Para 48 DMUs, as diferenças decorrem das dez DMUs $100 \%$ eficientes, e, portanto, empatadas na abordagem BCC tradicional. Em seis 
RUBEM, A.P.S; MOURA, A.L; SOARES DE MELLO, J.C.C.B; SANTOS, B.M.F.

Tabela 6. Resultados de eficiência e ordenação da abordagem proposta e da BCC tradicional

\begin{tabular}{|c|c|c|c|c|c|c|c|c|c|}
\hline \multirow[b]{2}{*}{$D M U$} & \multicolumn{4}{|c|}{ Eficiência } & \multirow[b]{2}{*}{$D M U$} & \multicolumn{4}{|c|}{ Eficiência } \\
\hline & Ordem & $\begin{array}{r}\text { Cluster }+ \text { CCR } \\
+ \text { compensação }\end{array}$ & Ordem & $B C C$ & & Ordem & $\begin{array}{c}\text { Cluster }+ \text { CCR } \\
+ \text { compensaçãao }\end{array}$ & Ordem & $B C C$ \\
\hline Abaete_10 & 54 & $10,2 \%$ & 32 & $30,7 \%$ & Abaete_08 & 68 & $7,1 \%$ & 36 & $28,1 \%$ \\
\hline Avianca_10 & 27 & $30,4 \%$ & 21 & $46,7 \%$ & Air Minas_08 & 50 & $11,4 \%$ & 53 & $21,0 \%$ \\
\hline Azul_10 & 11 & $50,8 \%$ & 12 & $87,4 \%$ & Gol/Varig_08 & 16 & $46,6 \%$ & 13 & $78,1 \%$ \\
\hline Gol/Varig_10 & 13 & $49,1 \%$ & 1 & $100,0 \%$ & Meta_08 & 29 & $26,6 \%$ & 27 & $36,5 \%$ \\
\hline Meta_10 & 42 & $14,0 \%$ & 42 & $25,1 \%$ & NHT_08 & 58 & $9,4 \%$ & 58 & $18,4 \%$ \\
\hline $\mathrm{NHT}_{-} 10$ & 69 & $4,9 \%$ & 69 & $12,6 \%$ & Oceanair_08 & 38 & $18,6 \%$ & 35 & $29,4 \%$ \\
\hline Noar_10 & 4 & $66,3 \%$ & 18 & $51,0 \%$ & Pantanal_08 & 32 & $23,9 \%$ & 61 & $18,1 \%$ \\
\hline Pantanal_10 & 22 & $34,9 \%$ & 45 & $23,6 \%$ & Passaredo_08 & 24 & $32,5 \%$ & 26 & $37,9 \%$ \\
\hline Passaredo__10 & 7 & $59,0 \%$ & 24 & $38,8 \%$ & Puma_08 & 70 & $3,1 \%$ & 68 & $14,1 \%$ \\
\hline Puma_10 & 1 & $100,0 \%$ & 1 & $100,0 \%$ & Rico_08 & 45 & $13,2 \%$ & 71 & $10,8 \%$ \\
\hline Rico_10 & 8 & $57,5 \%$ & 23 & $40,1 \%$ & Sete_08 & 57 & $9,8 \%$ & 41 & $25,1 \%$ \\
\hline Sete_10 & 49 & $11,5 \%$ & 50 & $21,9 \%$ & TAF_08 & 52 & $10,4 \%$ & 65 & $15,6 \%$ \\
\hline Sol 10 & 37 & $20,2 \%$ & 16 & $52,9 \%$ & TAM 08 & 10 & $52,4 \%$ & 1 & $100,0 \%$ \\
\hline TAM_10 & 12 & $50,3 \%$ & 1 & $100,0 \%$ & Team_08 & 48 & $11,8 \%$ & 52 & $21,2 \%$ \\
\hline Team_10 & 51 & $11,1 \%$ & 49 & $22,1 \%$ & Total_08 & 60 & $9,0 \%$ & 72 & $10,5 \%$ \\
\hline Total_10 & 43 & $13,9 \%$ & 39 & $26,8 \%$ & Trip_08 & 30 & $24,9 \%$ & 25 & $38,2 \%$ \\
\hline Trip_10 & 33 & $23,7 \%$ & 28 & $35,8 \%$ & Webjet_08 & 20 & $37,9 \%$ & 15 & $58,2 \%$ \\
\hline Webjet_10 & 19 & $39,2 \%$ & 14 & $71,5 \%$ & & & & & \\
\hline Abaete_09 & 64 & $8,5 \%$ & 34 & $29,4 \%$ & Abaete_07 & 53 & $10,3 \%$ & 44 & $23,7 \%$ \\
\hline AirMinas_09 & 61 & $8,9 \%$ & 64 & $15,9 \%$ & AirMinas_07 & 65 & $7,6 \%$ & 63 & $17,1 \%$ \\
\hline Avianca_09 & 34 & $22,3 \%$ & 29 & $33,7 \%$ & Gol_07 & 4 & $66,3 \%$ & 1 & $100,0 \%$ \\
\hline Azul_09- & 4 & $66,3 \%$ & 1 & $100,0 \%$ & Mega_07 & 21 & $35,8 \%$ & 1 & $100,0 \%$ \\
\hline Gol/V̄arig_09 & 18 & $42,6 \%$ & 1 & $100,0 \%$ & Meta_07 & 39 & $17,6 \%$ & 37 & $27,6 \%$ \\
\hline Meta_09 & 46 & $13,1 \%$ & 46 & $23,4 \%$ & NHT_07 & 62 & $8,6 \%$ & 55 & $19,6 \%$ \\
\hline NHT_09 & 67 & $7,2 \%$ & 67 & $14,9 \%$ & Oceanair_07 & 40 & $16,9 \%$ & 40 & $26,1 \%$ \\
\hline Pantanal_09 & 31 & $24,6 \%$ & 56 & $18,9 \%$ & Pantanal_07 & 28 & $28,0 \%$ & 54 & $20,7 \%$ \\
\hline Passaredo_09 & 3 & $68,8 \%$ & 20 & $46,8 \%$ & Passaredo_07 & 25 & $32,2 \%$ & 22 & $42,0 \%$ \\
\hline Puma_09 & 73 & $0,03 \%$ & 70 & $11,8 \%$ & Puma_07 & 66 & $7,2 \%$ & 60 & $18,1 \%$ \\
\hline Rico_09 & 72 & $0,7 \%$ & 73 & $2,2 \%$ & Rico_07 & 26 & $31,8 \%$ & 51 & $21,8 \%$ \\
\hline Sete_09 & 59 & $9,2 \%$ & 48 & $22,4 \%$ & Sete_07 & 63 & $8,6 \%$ & 43 & $23,8 \%$ \\
\hline Sol_09 & 35 & $21,8 \%$ & 1 & $100,0 \%$ & TAF_07 & 47 & $12,1 \%$ & 59 & $18,4 \%$ \\
\hline TAF_09 & 14 & $47,9 \%$ & 31 & $31,0 \%$ & TAM__07 & 9 & $56,3 \%$ & 1 & $100,0 \%$ \\
\hline TAM_09 & 15 & $46,8 \%$ & 11 & $91,1 \%$ & Team_07 & 71 & $2,7 \%$ & 62 & $17,4 \%$ \\
\hline Team_09 & 41 & $15,1 \%$ & 38 & $27,3 \%$ & Total_07 & 55 & $9,9 \%$ & 66 & $15,0 \%$ \\
\hline Total_09 & 44 & $13,4 \%$ & 47 & $22,5 \%$ & Trip_07 & 17 & $44,3 \%$ & 33 & $29,9 \%$ \\
\hline Trip_09 & 36 & $21,2 \%$ & 30 & $32,2 \%$ & Varig_07 & 56 & $9,9 \%$ & 57 & $18,6 \%$ \\
\hline Webjet 09 & 23 & $33,6 \%$ & 17 & $52,2 \%$ & Webjet 07 & 2 & $72,5 \%$ & 19 & $47,7 \%$ \\
\hline
\end{tabular}

casos, as ordenações finais em ambas as abordagens coincidem: Meta, em 2010 e 2009; NHT, nos anos de 2008, 2009 e 2010; e Oceanair, em 2007. Contudo, é possível identificar alguns casos em que diferenças nas ordenações se sobressaem: por exemplo, Abaeté, em 2009 e 2008; Pantanal, em 2008; e Sol, em 2009.

\section{CONCLUSÕES}

O objetivo deste artigo era realizar uma análise os resultados de eficiência obtidos por meio uma abordagem $\mathrm{BCC}$ tradicional em comparação à metodologia aqui proposta, que representa uma forma alternativa de se lidar com diferenças na escala de operação das DMUs.

A proposta metodológica aqui apresentada combina clusterização ex-ante das DMUs ao modelo CCR, e compensação ex-post das diferenças de escala entre os clusters.

Para a consecução do objetivo, ambas abordagens foram aplicadas buscando avaliar o desempenho operacional das empresas brasileiras de transporte aéreo de passageiros, no período de 2007 a 2010, no que se refere, estritamente, ao uso da frota.
Os resultados evidenciam que a abordagem proposta discriminou melhor as DMUs, tendo redundado em apenas uma empresa eficiente. Além disso, ficou claro que as DMUs classificadas como eficiente no BCC tradicional, por razões estritamente matemáticas, estão bem longe de serem de fato eficientes.

Não obstante, a proposta pareceu penalizar os resultados de eficiência, uma vez, em $82 \%$ dos casos, as pontuações foram menores que na abordagem BCC tradicional. Surpreendentemente, em $66 \%$ dos casos, as ordenações finais das duas abordagens foram bastante semelhantes, desconsiderando-se os empates do BCC tradicional.

Pode-se concluir que, em relação ao modelo BCC tradicional, a abordagem proposta apresenta a vantagem de evitar as classificações inadequadas de DMUs eficientes, bem como outros inconvenientes daquele modelo (vide Subseção 2.1). Com isso, a proposta evidencia maior capacidade de discriminação entre as DMUs que a abordagem BCC usual, uma vez que resulta em uma quantidade menor de unidades eficientes. 
Uma sugestão de trabalho futuro compreende a análise comparativa da metodologia aqui proposta com a abordagem que integra a clusterização dinâmica ao modelo CCR (Golany e Thore, 1997).

\section{AGRADECIMENTOS}

Ao CNPq, pelo apoio financeiro.

\section{REFERÊNCIAS}

Ali, A.I. (1993) Streamlined computation for data envelopment analysis. European Journal of Operational Research, v. 64, n. 1, p. 61-67. DOI: $10.1016 / 0377-2217(93) 90008-B$.

ANAC (2007) Anuários estatísticos do transporte aéreo 2007. Disponível em: www.anac.gov.br/ [acesso em: 20 de maio de 2014].

ANAC (2008) Anuários estatísticos do transporte aéreo 2008. Disponível em: www.anac.gov.br/ [acesso em: 20 de maio de 2014].

ANAC (2009) Anuários estatísticos do transporte aéreo 2009. Disponível em: www.anac.gov.br/ [acesso em: 20 de maio de 2014].

ANAC (2010) Anuários estatísticos do transporte aéreo 2010. Disponível em: www.anac.gov.br/ [acesso em: 20 de maio de 2014].

Angulo-Meza, L.; Biondi Neto, L.; Soares de Mello, J. C. C. B. e Gomes, E. G. (2005) ISYDS - Integrated System for Decision Support (SIAD - Sistema Integrado de Apoio a Decisão): A software package for data envelopment analysis model. Pesquisa Operacional, v. 25, n. 3, p. 493503. DOI: 10.1590/S0101-74382005000300011.

Jain, A. K. (2010) Data clustering: 50 years beyond Kmeans. Pattern Recognition Letters, v. 31, n. 8, p. 651666. DOI:10.1016/j.patrec.2009.09.011.

Banker, R. D.; Charnes, A. e Cooper, W. W. (1984) Some models for estimating technical scale inefficiencies in data envelopment analysis. Management Science, v. 30, n. 9, p. 1078-1092. DOI: 10.1287/mnsc.30.9.1078.

Banker, R. D.; Charnes, A.; Cooper, W. W.; Swarts, J. e Thomas, D. (1989) An introduction to data envelopment analysis with some models and their uses. Research in Governmental and Non-Profit Accounting, v. 5, p. 125-163.

Bertoloto, R. F. e Soares de Mello, J. C. C. B. (2011) Eficiência de portos e terminais privativos brasileiros com características distintas. Journal of Transport Literature, v. 5, n. 2, p. 4-21.

Correia, T. C. V. D.; Soares de Mello, J. C. C. B. e Angulo-Meza, L. (2011) Assessment of Brazilian airlines technical efficiency: a study using data envelopment analysis and fuzzy sets, Produção, v. 1, n. 4, p. 676-683. DOI: $10.1590 /$ S0103-65132011005000049.

Golany, B. e Thore, S. (1997) Restricted best practice selection in DEA: An overview with a case study evaluating the socio-economic performance of nations. Annals of $\mathrm{Op}$ erations Research, v. 73, p. 117-140.

DOI: 10.1023/A:1018916925568.

Gomes, E. G.; Abreu, U. G. P.; Soares de Mello, J. C. C. B.; Carvalho, T. B. e Zen, S. (2012a) DEA performance evaluation of livestock systems in Brazil. In: Data envelopment analysis and its applications to management, Charles, V. e Kumar, M. (eds.) Cambridge Scholars Publishing, Newcastle upon Tyne, Reino Unido, p. 224-238.

Gomes, E. G.; Soares de Mello, J. C. C. B. e Freitas, A. C. R. (2012b) Efficiency measures for a non-homogeneous group of family farmers. Pesquisa Operacional, v. 32, n. 3, p. 561-574.

Gomes Júnior, S. F.; Rubem, A. P. S.; Soares de Mello, J. C. C. B. e Angulo-Meza, L. (2015) Evaluation of Brazilian airlines nonradial efficiencies and targets using an alternative DEA approach. International Transactions in Operational Research. DOI: 10.1111/itor.12162.

Gomes Júnior, S. F.; Soares de Mello, J. C. C. B. e Angulo-Meza, L. (2013) DEA nonradial efficiency based on vector properties. International Transactions in Operational Research, v. 20, n. 3, p. 341-364. DOI: 10.1111/itor.12000.

MacQueen, J. B. (1967) Some Methods for classification and Analysis of Multivariate Observations. In: Proceedings of 5th Berkeley Symposium on Mathematical Statistics and Probability, University of California Press, California, CA, Estados Unidos, v. 1, n. 14, p. 281-297.

Podinovski, V. V. e Thanassoulis, E. (2007) Improving discrimination in data envelopment analysis: Some practical suggestions. Journal of Productivity Analysis, v. 28, n. 1-2, p. 117-126. DOI 10.1007/s11123-007-0042-x.

Silveira, J. Q.; Soares de Mello, J. C. C. B. e AnguloMeza, L. (2012a) Brazilian airlines efficiency evaluation using a data envelopment analysis (DEA) and multiobjective linear programming hybrid model. Ingeniare, v. 20, n. 3, p. 331-342.

Silveira, J. Q.; Soares de Mello, J. C. C. B., e AnguloMeza, L. (2012b) Identificação de benchmarks e anti-benchmarks para companhias aéreas usando modelos DEA e fronteira invertida. Produção, v. 22, n. 4, p. 788-795.

Soares de Mello, J. C. C. B.; Angulo-Meza, L.; Gomes, E. G.; Serapião, B. P. e Lins, M.P E. (2003) Análise de envoltória de dados no estudo da eficiência e dos benchmarks para companhias aéreas brasileiras. Pesquisa Operacional, v. 23, n. 2, p. 325-345. DOI: $10.1590 / \mathrm{S} 0101-74382003000200005$. 
Soares de Mello, J. C. C. B.; Angulo Meza, L.; Silveira, J. Q. e Gomes, E. G. (2013) About negative efficiencies in cross evaluation BCC input oriented models. European Journal of Operational Research, v. 229, n. 3, p. 732-737. DOI:10.1016/j.ejor.2013.02.020.

Soares de Mello, J. C. C. B.; Gomes, E. G.; Angulo-Meza, L.; Biondi Neto, L.; Abreu, U. G. P.; Carvalho, T. B. e Zen, S. (2012) Ex-post clustering of Brazilian beef cattle farms using SOMs and cross-evaluation DEA models, Applications of self-organizing maps, Ed. Intech.

DOI: $10.5772 / 51324$.

Soares de Mello, P. H. C.; Soares de Mello, J. C. C. B. e Angulo-Meza, L. (2009) Misunderstandings due to a codeshare between two Brazilian airlines in Rio de Janeiro international airport. Rio's International Journal on sciences of industrial and systems engineering and management, v. 3, n. 1, p. sp091-02.

Steinhaus, H. (1957) Sur la division des corps matériels en parties. Bulletin de L'Academie Polonaise des Sciences, v. 4, n. 12 , p. $801-804$. 